C.M.B. MONTEIRO ${ }^{1}$

L.M.P. FERNANDES ${ }^{1}$

J.A.M. LOPES ${ }^{1,2}$

J.F.C.A. VELOSO ${ }^{1,3}$

J.M.F. DOS SANTOS 1 ,

\section{Detection of VUV photons with large-area avalanche photodiodes}

\author{
${ }^{1}$ Physics Department, University of Coimbra, 3004-516 Coimbra, Portugal \\ ${ }^{2}$ Instituto Superior de Engenharia de Coimbra, Rua Pedro Nunes, Apartado 4065, 3030-199 Coimbra, Portugal \\ ${ }^{3}$ Physics Department, University of Aveiro, 3810-193 Aveiro, Portugal
}

Received: 5 January 2005 / Revised version: 3 May 2005 Published online: 21 June 2005 • () Springer-Verlag 2005

ABSTRACT The room-temperature response of large-area avalanche photodiodes (LAAPDs) to 128- and 172-nm light pulses is investigated. The minimum detectable number of photons, which can produce a signal just above the noise level, is found to be around 1300 and 600 photons, respectively. The LAAPD relative statistical fluctuations in the detection of 15000 photons of $128 \mathrm{~nm}$ and 25500 photons of $172 \mathrm{~nm}$ were found to be about $3.9 \%$ and $2.2 \%$, respectively. Both the minimum detectable number of photons and statistical fluctuations do not depend on the photon wavelength, but rather on the number of charge carriers produced by the light pulse in the LAAPD. For these light levels, good LAAPD performance is already achieved for gains as low as 30 to 60 .

PACS 07.60.-j; 42.70.Gi; 42.79.-e

1

\section{Introduction}

In recent years, significant advances in the development of large-area avalanche photodiodes (LAAPDs) triggered the study and the characterization of different commercially available avalanche photodiodes (APDs) (e.g. Hamamatsu [1, 2], EG\&G [2, 3], API [4, 5], and RMD [6, 7]). Although applications to direct X-ray detection have been investigated [4, 8-10], LAAPDs have been used mainly as optical photosensors coupled to scintillation detectors for X-, $\gamma$-ray, and particle detection, such as in the electromagnetic calorimeter of the Compact Muon Solenoid (CMS) detector $[1,2,8]$, in gas proportional scintillation counters [11, 12], and in Positron Emission Tomography (PET) $[3,13,14]$ and nuclear physics $[5,6]$ instrumentation. Nevertheless, these devices can be applied to photon detection in other areas of optics.

It has been demonstrated that LAAPDs can replace photomultiplier tubes (PMTs) with advantages, delivering similar performances. When compared to the PMTs, LAAPDs are much more compact, present much less power consumption, have a straightforward operation, can operate in intense magnetic fields and have higher quantum efficiencies. On the

Fax: +351-239-829158, E-mail: jmf@gian.fis.uc.pt other hand, their low gains and reduced active areas present the main drawbacks of LAAPDs. In addition, low-energy $\mathrm{X}$-ray detection techniques with APDs were developed to measure the charge carriers produced in light measurements using X-rays as a reference, resulting in a straightforward process to evaluate the number of photons interacting in the photodiode.

More recently, API has developed window-less LAAPDs with a spectral response that extends down to the vacuumultraviolet (VUV) region $(\sim 120 \mathrm{~nm})$ [15]. Enhanced quantum efficiency is the result of maximizing the quantum yield and transmittance, while reducing recombination, through the selection of an optimized thickness of silicon dioxide as the antireflection layer. The high-quality silicon surface also reduces losses from recombination of charge carriers. These LAAPDs can replace PMTs or CsI-based photosensors in applications where the light level allows the use of low-gain photosensors [11, 12, 16-19]. For example, the detection of primary and secondary scintillation in rare gases is not only used in gas scintillation X-ray detectors [11, 12], but also in many other types of radiation detectors, which have been investigated recently [16-19].

Different studies have proved that the LAAPD response characteristics for VUV are different from those for visible light, used to determine most of the characteristics of the photodiodes [20-22]. The X-ray-to-photon detection nonlinearity, the sensitivity to magnetic fields and the relative variation of gain with temperature have been measured for VUV, presenting higher values when compared to visiblelight detection. This is due to the difference in the average depth of photon interaction $(\sim 1 \mu \mathrm{m}$ and $\sim 5 \mathrm{~nm}$ for $520-\mathrm{nm}$ and 172-nm photons, respectively [1,23]): VUV photons interact mainly within the first atomic layers, where the electric field is weaker, resulting in higher diffusion of charge carriers that can be lost to the surface boundary and to impurities.

In this work we investigate the response characteristics of a 'Deep-UV 500 window-less series' LAAPD from API to the argon and xenon electroluminescence or secondary scintillation light (a narrow continuum peaking at $\sim 128$ and $\sim 172 \mathrm{~nm}$, respectively, with a full-width-at-half-maximum of $\sim 10 \mathrm{~nm}$ in both cases [24]), in the lower limit of the LAAPD spectral response. The LAAPD gain is studied, as well as the X-ray-to-photon non-linearity, the minimum 


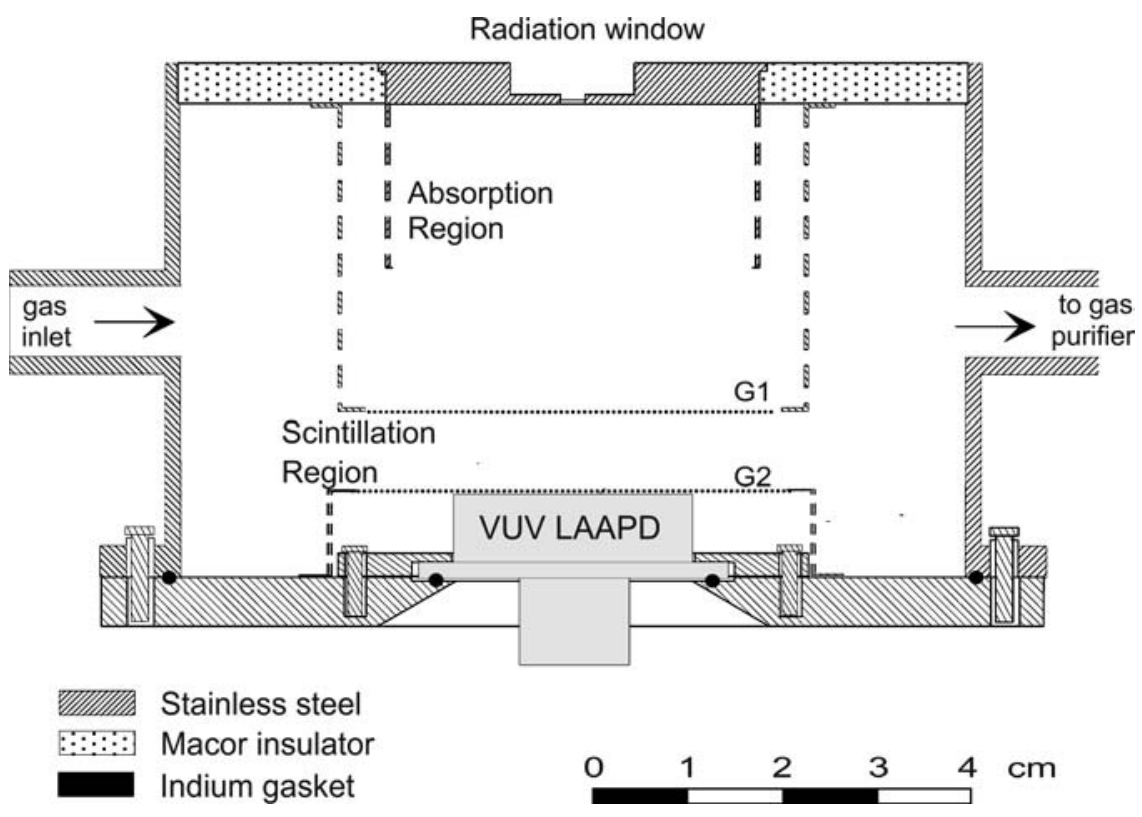

FIGURE 1 The schematic of the GPSC instrumented with a large-area APD photosensor

detectable number of photons and the overall statistical fluctuations associated with photon detection, as a function of gain.

\section{2}

\section{Experimental set-up}

The LAAPD was placed inside a gas proportional scintillation counter (GPSC) as a photosensor, substituting for the PMT, Fig. 1. In this type of detector, pure rare gases or their mixtures are used as scintillators.

Detailed theory and operation of GPSCs are described in Ref. [25] and references therein. Incident X-rays interact primarily in the drift region, Fig. 1, where the electric field intensity is kept below the gas-excitation threshold. The primary electron cloud resulting from the X-ray interaction drifts into the scintillation region, where the electric field intensity is higher, but kept below, or around, the gas-ionization threshold. In this way, upon traversing the scintillation region, the primary electrons gain enough energy from the electric field to excite the gas, producing a secondary scintillation pulse, without further ionizing the gas.

The GPSC used in this work, Fig. 1, has been described in earlier publications $[11,12]$. The radiation window and its focusing electrode are operated at negative voltage, while the wire mesh G2, the LAAPD enclosure, and the detector body are maintained at ground potential. The voltage difference between $\mathrm{G} 1$ and the radiation window determines the electric field in the drift region, while G1 voltage determines the electric field in the scintillation region. The detector was filled with gas, either pure argon at $1.5 \times 10^{5} \mathrm{~Pa}$ or pure xenon at $1.1 \times 10^{5} \mathrm{~Pa}$, which was continuously purified through nonevaporable getters.

Throughout this experimental work, the reduced electric field (i.e. the electric field divided by the gas pressure, $E / p$ ) in the scintillation region was kept constant at $6.6 \mathrm{~V} \mathrm{~m}^{-1} \mathrm{~Pa}^{-1}$ and $8.6 \mathrm{~V} \mathrm{~m}^{-1} \mathrm{~Pa}^{-1}$ for argon and xenon filling, respectively. Under these conditions, for each $5.9-\mathrm{keV}$ X-ray interac- tion in the GPSC drift region, an average number of about 15000 VUV photons, resulting from the argon secondary scintillation, hit the photodiode [12], while for the xenon scintillation this number is about 25500 photons [11]. The $\mathrm{X}$-ray interaction rate in the gas was maintained at about a few hundred counts per second.

The LAAPD has a 16-mm active diameter and is placed just below grid G2, i.e. just below the scintillation region. It is vacuum sealed by compressing its enclosure against the detector body with an indium ring. It is biased with a variable voltage for operation at different gains. The APD signals were fed through a low-noise $1.5-\mathrm{V} / \mathrm{pC}$ charge preamplifier to an amplifier with $2-\mu$ s shaping time and were pulse analysed with a multichannel analyser. For pulse amplitude and energy resolution measurements the pulse-height distributions were fitted to Gaussian functions superimposed on a linear background, from which the centroid and the full-width-at-half-maximum (FWHM) were determined.

\section{$3 \quad$ Experimental results and discussion}

Figure 2 depicts a typical pulse-height distribution obtained with the Ar- (Fig. 2a) and Xe-GPSC (Fig. 2b) when irradiated with $5.9-\mathrm{keV}$ X-rays from ${ }^{55} \mathrm{Fe}$, with the $K_{\beta}$-line filtered through a chromium film (for the argon-filling case). Approximately $10 \%$ of the $5.9-\mathrm{keV}$ X-rays are transmitted through the $3.3-\mathrm{cm}$ argon depth and $40 \%$ of these are absorbed in the depletion region of the APD. For xenon filling, the 5.9$\mathrm{keV} \mathrm{X}$-rays are fully absorbed in the gas and do not reach the LAAPD. The salient features of the pulse-height distributions include the argon or xenon scintillation peaks resulting from the full absorption of the 5.9-keV X-rays in the gas and from the subsequent escape of the argon or xenon fluorescence, the 5.9-keV X-ray peak from direct absorptions in the LAAPD (for the argon-filling case) and the electronic noise tail in the low-energy limit. The spectra obtained with the argon GPSC 

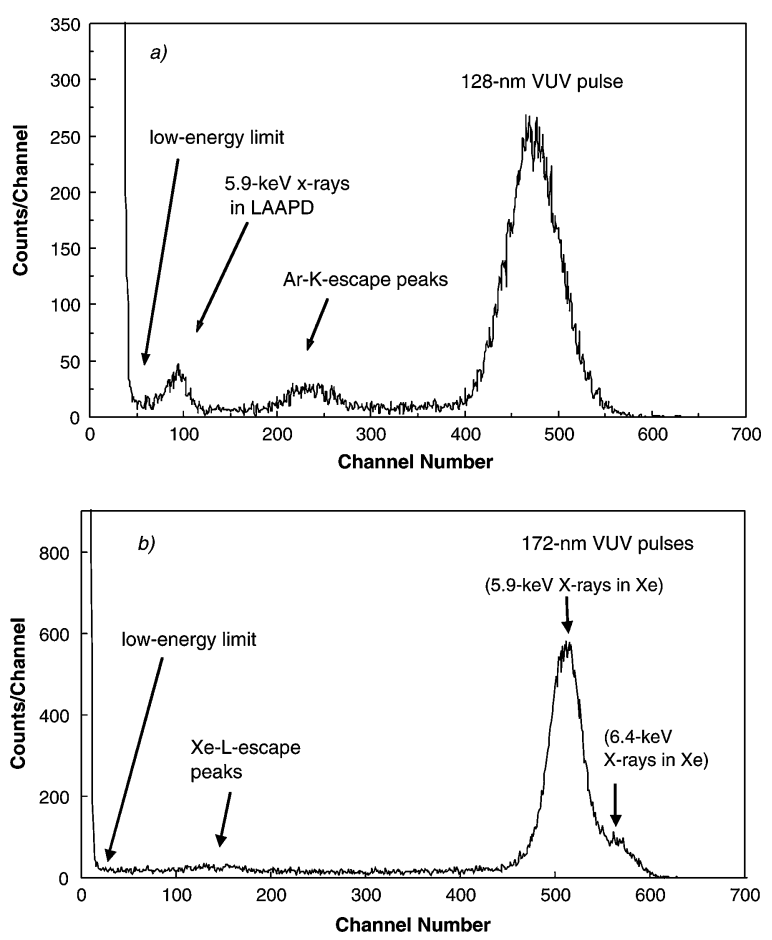

FIGURE 2 a Pulse-height distribution resulting from both argon VUVscintillation pulses and 5.9-keV X-ray interactions in the LAAPD, for gains around 40. b Pulse-height distribution resulting from xenon VUV-scintillation pulses in the LAAPD, for gains around 60

allow a direct comparison of the relative amplitudes from the argon scintillation and from the X-ray interaction in the APD, for each value of the APD biasing voltage.

As seen from Fig. 2a, the argon scintillation pulse deposits in the LAAPD an amount of energy similar to what would be deposited by a $\sim 30-\mathrm{keV}$ X-ray interaction in the photodiode. This is consistent with a LAAPD spectral sensitivity of $51 \mathrm{~mA} / \mathrm{W}$ (corresponding to an average of 0.50 free electrons produced in the photodiode per incident VUV photon), which is in agreement with the manufacturer's specification.

In Fig. 3 we depict the LAAPD gain for both argonscintillation and 5.9-keV X-ray pulses in the LAAPD as a

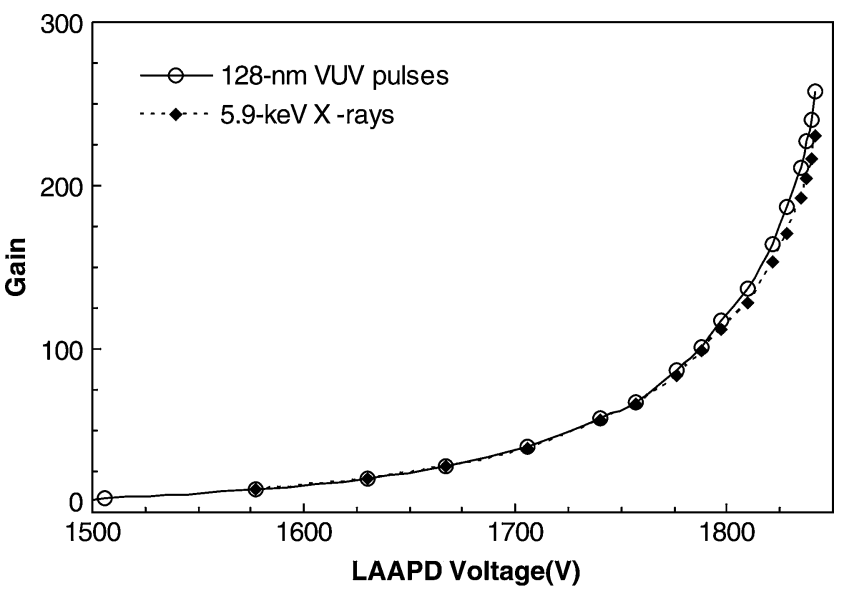

FIGURE 3 LAAPD gains for the 128-nm scintillation pulses and 5.9-keV $\mathrm{X}$-ray interactions in the LAAPD as a function of its biasing voltage. The lines serve only to guide the eyes

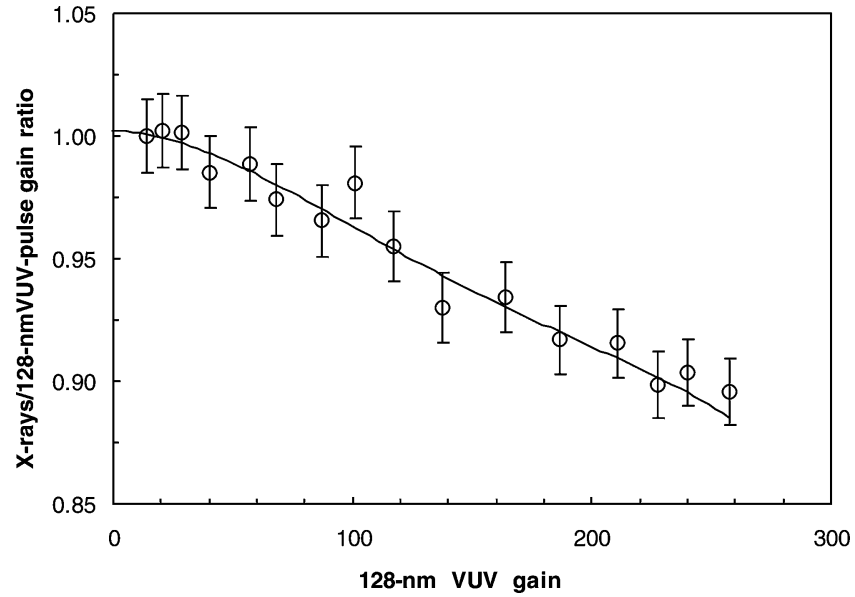

FIGURE 4 Ratio between LAAPD gains for 5.9-keV X-rays and for 128$\mathrm{nm}$ scintillation pulses as a function of the VUV-scintillation gain. The line serves only to guide the eyes

function of LAAPD biasing voltage. Absolute gains were obtained by normalizing the gain to 13.8 for a biasing voltage of $1577 \mathrm{~V}$, following the photodiode data sheet for low gains [15]. X-rays have always been used to determine the number of charge carriers produced in light measurements by comparison of the relative pulse amplitudes. The comparison is valid provided that strict linearity is assumed between the initial number of charge carriers and the resulting pulse amplitude, throughout the range of avalanche multiplication gain.

Non-linearities have been reported at high current densities and attributed to space-charge effects, reduced local electric fields, and heating in the avalanche region that occur for $\mathrm{X}$-ray interactions at high gains $[4,20]$. While charge carriers produced by X-rays are concentrated in a very small volume of the APD, within a few microns, for light measurements they are produced within a much larger region.

The X-ray-to-scintillation-gain ratio is depicted in Fig. 4. Non-linear effects are less than 2, 4, and $10 \%$ for gains below 50, 100, and 240, respectively. They are higher than those obtained for 172-nm photons and for visible light [4, 20]. Nevertheless, the non-linearities observed in this type of photodiode are smaller than those observed with other APD types [4].

In Fig. 5 we present the minimum number of detectable photons (MDP) for argon and xenon secondary scintillation, defined as the number of photons that would deposit, in the LAAPD, an amount of energy equivalent to the onset of the electronic noise tail. The MDP shows a similar trend in both cases: it is approximately constant being, for the present conditions, about 1300 and 600 photons for 128-nm and 172-nm VUV light pulses, for gains above $\sim 60$ and $\sim 40$, respectively, increasing significantly as the gain drops below these values and the signal approaches the noise level.

As expected, the MDP for 172-nm photons is about half of that for 128-nm photons, achieving the lowest values for lower gains, reflecting the higher spectral sensitivity of the LAAPD for $172 \mathrm{~nm}(\sim 150 \mathrm{~mA} / \mathrm{W}$, corresponding to an average of 1.1 free electrons produced in the photodiode per incident VUV photon [15]). In fact, it is the number of primary charge carriers produced by the light pulse that will define 


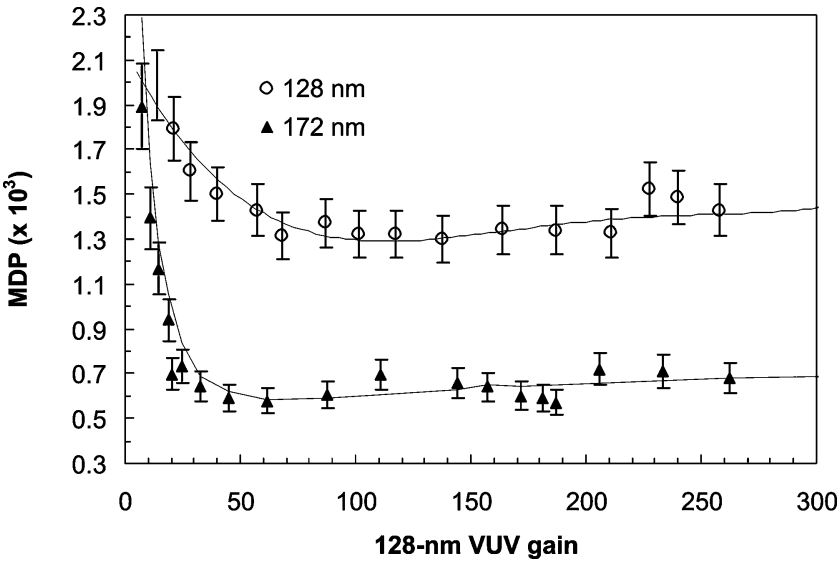

FIGURE 5 Minimum number of detectable 128-nm and 172-nm VUV photons as a function of LAAPD gain. The lines serve only to guide the eyes

the corresponding signal amplitude and signal-to-noise ratio. The results obtained with this LAAPD for MDP at $172 \mathrm{~nm}$ are lower than those obtained with the Peltier-cooled LAAPD in Ref. [22] ( 1100 photons). The difference may be attributed to the differences in the LAAPD's dark currents, which limit the electronic noise and, thus, the MDP. Nevertheless, the obtained MDP can be reduced by up to a factor of two by cooling the temperature of the photodiode to temperatures below $0^{\circ} \mathrm{C}$ [26].

The statistical fluctuations associated with the detection of VUV light in the LAAPD may be estimated from the measured energy resolution of the pulse-height distributions of 5.9-keV full absorptions in the gas. The energy resolution of a conventional GPSC is determined by the statistical fluctuations occurring in the primary ionization processes in the gas, in the production of the VUV scintillation photons, and in the photosensor. Since the statistical fluctuations associated with the scintillation processes are negligible when compared to those associated with the primary electron-cloud formation, in the gas, and those associated with the scintillation detection, in the photosensor, the energy resolution, $R$, of the GPSC, for an X-ray energy $E_{\mathrm{x}}$, is given by [25]

$R=2.355 \sqrt{\frac{F}{N_{\mathrm{e}}}+\left(\frac{\Delta E}{E}\right)^{2}}=2.355 \sqrt{\frac{F w}{E_{\mathrm{x}}}+\left(\frac{\Delta E}{E}\right)^{2}}$,

where $N_{\mathrm{e}}$ is the average number of primary electrons produced in the gas by the X-rays, $F$ is the Fano factor, $w$ is the average energy to create a primary electron in the gas, and $E$ is the energy deposited by the VUV radiation in the photosensor.

The statistical fluctuations associated with the VUVphoton detection can, thus, be obtained by

$\frac{\Delta N_{\mathrm{UV}}}{N_{\mathrm{UV}}}=\frac{\Delta E}{E}=\sqrt{\left(\frac{R}{2.355}\right)^{2}-\frac{F w}{E_{\mathrm{x}}}}$.

In our case, $E_{\mathrm{x}}$ is $5.9 \mathrm{keV}, w=26.4 \mathrm{eV}$, and $F=0.30$ for argon, while for xenon $w=22.4 \mathrm{eV}$ and $F=0.17$. The relative statistical fluctuations associated with VUV detection of 15000 photons of $\sim 128 \mathrm{~nm}$ and of 25500 photons of $\sim 172 \mathrm{~nm}$ VUV-light pulses, as a function of gain, are depicted in Fig. 6. The LAAPD relative uncertainty decreases

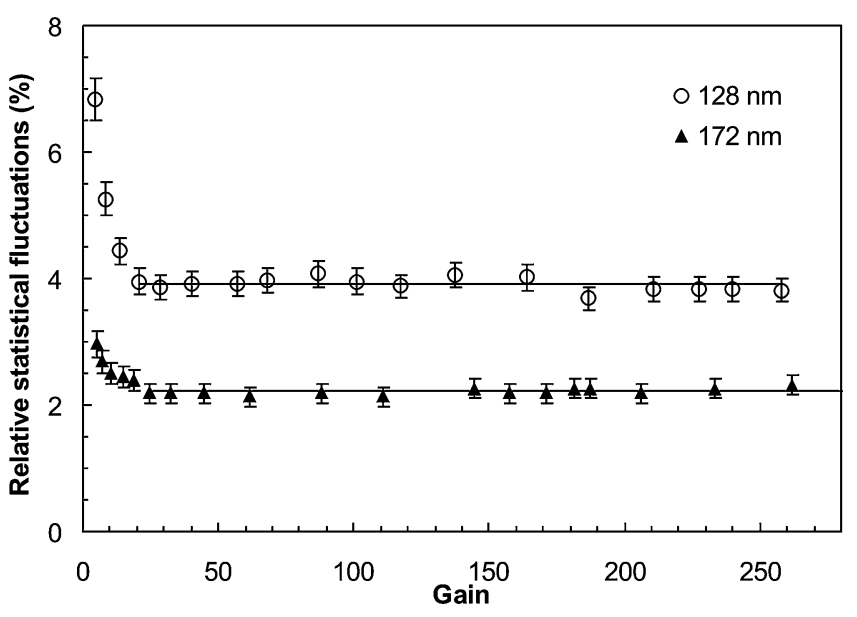

FIGURE 6 Relative statistical fluctuations associated with VUV detection of 15000 photons of $\sim 128$-nm and of 25500 photons of $\sim 172$-nm VUV light pulses as a function of LAAPD gain. The lines serve only to guide the eyes

rapidly with the onset of gain, stabilizing for gains above $\sim 30$ and reaching values of $3.9 \%$ and $2.2 \%$, respectively, for the above cases. This difference is consistent with the dependence of the LAAPD resolution on the inverse of the square root of the number of charge carriers produced in the photodiode [4] and it reflects not only the difference in the number of photons involved in each case, but also the difference in the respective quantum efficiency. These values can be reduced by cooling the photodiode operating temperature to values around $0^{\circ} \mathrm{C}[26]$.

From Figs. 5 and 6 we conclude that, for the detection of the light levels of 128- and 172-nm photons studied in this work, i.e. around $2 \times 10^{4}$ photons, best performance characteristics are achieved for gains of about 60 and 40, respectively. However, gains as low as 30 and 20, respectively, are sufficient to achieve a nearly optimum performance, i.e. without presenting significant degradation of MDP and resolution, as can be seen from Figs. 5 and 6. For lower light levels, higher gains will be needed to pull the signal of the light pulse out of the noise and achieve the best possible performance.

Presently, the LAAPD manufacturing technology is well established and quite good reproducibility is obtained. Thus, it is expected that the observed behaviour for individual LAAPDs is representative for any of these devices [M. Szawlowsky, Advanced Photonics Inc. (2002), private communication].

\section{Conclusions}

The LAAPD is a suitable device for the detection of VUV light pulses of photons down to about $120 \mathrm{~nm}$. Using $\mathrm{X}$-rays as a reference, it is straightforward to determine the number of the VUV photons present in the light pulse. The LAAPD is not suitable for single-photon detection and VUVphoton spectrometry. Nevertheless, it can be applied to synchrotron radiation in VUV-photon detection and to other areas of optics, where the light levels are adequate for its use. To the best of our knowledge, there is no other LAAPD type sensitive 
to the VUV region below $200 \mathrm{~nm}$. PMTs present a sensitivity range down to $115 \mathrm{~nm}$ (with $\mathrm{MgF}_{2}$ windows), gains above $10^{7}$, and dark currents below a few $\mathrm{nA}$, instead of a few hundred nA for the LAAPDs, and are suitable for single-photon detection. However, the photodiode compactness, reduced power consumption, simple operation, and straightforward photon calibration are significant advantages over PMTs.

The detection limit of the LAAPD, in terms of number of photons in the pulse, can be as low as a few hundred photons: under the conditions of our experiment the minimum number of photons that can be detected with the LAAPD is about 1300 and 600 for 128 and $172 \mathrm{~nm}$, respectively. The relative statistical fluctuations associated with VUV detection of 15000 photons of $\sim 128 \mathrm{~nm}$ and of 25500 photons of $\sim 172 \mathrm{~nm}$ VUV-light pulses are $3.9 \%$ and $2.2 \%$, respectively. The experimental results show that both quantities do not depend on the photon wavelength, but rather on the number of charge carriers produced by the light pulse in the LAAPD, in opposition to non-linearity effects and to the behaviour under intense magnetic fields. For the above two cases, photodiode gains as low as 30 to 60 are enough to obtain the best performances. However, higher gains will be necessary in applications were the amount of light to be detected is lower.

ACKNOWLEDGEMENTS This work was performed in the Instrumentation Centre of the Faculty of Sciences and Technology of the University of Coimbra and it was supported by Project No. POCTI/FIS/60534/2004.

\section{REFERENCES}

1 K. Deiters, Y. Musienko, S. Nicol, B. Patel, D. Renker, S. Reucroft, R. Rusak, T. Sakhelashvilli, J. Swain, P. Vikas, Nucl. Instrum. Methods A 442, 193 (2000)

2 A. Karar, Y. Musienko, J.Ch. Vanel, Nucl. Instrum. Methods A 428, 413 (1999)

3 R. Lecomte, C. Pepin, D. Rouleau, H. Dautet, R.J. McIntyre, D. McSwen, P. Webb, Nucl. Instrum. Methods A 423, 92 (1999)

4 M. Moszynski, M. Szawlowsky, M. Kapusta, M. Balcerzyk, Nucl. Instrum. Methods A 485, 504 (2002)

5 M. Moszynski, M. Szawlowsky, M. Kapusta, M. Balcerzyk, Nucl. Instrum. Methods A 497, 226 (2003)
6 R. Farrel, F. Olschner, K. Shah, M.R. Squillante, Nucl. Instrum. Methods A 387, 194 (1997)

7 R. Farrel, K. Shah, K. Vanderpuye, R. Graziaso, R. Myers, G. Entine, Nucl. Instrum. Methods A 442, 171 (2000)

8 J.P. Pansart, Nucl. Instrum. Methods A 387, 186 (1997)

9 L.M.P. Fernandes, J.A.M. Lopes, J.M.F. dos Santos, C.A.N. Conde, X-ray Spectrom. 30, 164 (2001)

10 L.M.P. Fernandes, A. Antognini, M. Boucher, C.A.N. Conde, O. Huot, P.E. Knowles, F. Kottmann, L. Ludhova, F. Mulhauser, R. Pohl, J.M.F. dos Santos, L.A. Schaller, D. Taqqu, J.F.C.A. Veloso, Spectrochim. Acta B 58, 2255 (2003)

11 J.A.M. Lopes, J.M.F. dos Santos, R.E. Morgado, C.A.N. Conde, IEEE Trans. Nucl. Sci. 48, 312 (2001)

12 C.M.B. Monteiro, J.A.M. Lopes, P.C.P.S. Simões, J.M.F. dos Santos, C.A.N. Conde, IEEE Trans. Nucl. Sci. 48, 1081 (2001)

13 B. Pichler, G. Böning, E. Lorenz, R. Mirzoyan, W. Pimpl, M. Schwaiger, S.I. Ziegler, IEEE Trans. Nucl. Sci. 45, 1298 (1998)

14 A. Ruru Chen, A. Fremout, S. Tavernier, P. Bruyndonckx, D. Clément, J.-F. Loude, C. Morel, Nucl. Instrum. Methods A 433, 637 (1999)

15 Application Notes, Advanced Photonix Inc., 1240 Av. Acaso, Camarillo, California, USA

16 V.N. Solovov, F. Neves, V. Chepel, M.I. Lopes, Nucl. Instrum. Methods A 504, 53 (2003)

17 L. Periale, V. Peskov, C. Iacobaeus, T. Francke, B. Lund-Jensen, N. Pavlopoulos, P. Picchi, F. Pietropaolo, Nucl. Instrum. Methods A 535, 517 (2004)

18 F. Sauli, T. Meinschad, L. Musa, L. Ropelewski, Photon detection and localization with GEM. Presented at 2004 Nuclear Science Symposium, Roma, Italy, 16-22 October 2004

19 M. Cwiok, W. Dominik, Z. Janas, A. Korgul, M. Miernik, M. Pfützner, M. Sawicka, A. Wasilewski, Optical time projection chamber for imaging of two-proton decay of ${ }^{45} \mathrm{Fe}$ nucleus. Presented at 2004 Nuclear Science Symposium, Roma, Italy, 16-22 October 2004

20 L.M.P. Fernandes, J.A.M. Lopes, C.M.B. Monteiro, J.M.F. dos Santos, R.E. Morgado, Nucl. Instrum. Methods A 478, 395 (2002)

21 L.M.P. Fernandes, A. Antognini, M. Boucher, C.A.N. Conde, O. Huot, P.E. Knowles, F. Kottmann, L. Ludhova, F. Mulhauser, R. Pohl, J.M.F. dos Santos, L.A. Schaller, D. Taqqu, J.F.C.A. Veloso, Nucl. Instrum. Methods A 498, 362 (2003)

22 J.A.M. Lopes, L.M.P. Fernandes, J.M.F. dos Santos, R.E. Morgado, C.A.N. Conde, Nucl. Instrum. Methods A 504, 331 (2003)

23 T.W. Barnard, M.M.I. Crockett, J.C. Ivaldi, P.L. Lundberg, D.A. Yates, P.A. Levine, D.J. Sauer, Anal. Chem. 65, 1231 (1993)

24 T. Takahashi, S. Himi, M. Suzuki, J. Ruan, S. Kubota, Nucl. Instrum. Methods A 205, 591 (1983)

25 J.M.F. dos Santos, J.A.M. Lopes, J.F.C.A. Veloso, P.C.P.S. Simões, T.H.V.T. Dias, F.P. Santos, P.J.B.M. Rachinhas, L.F.R. Ferreira, C.A.N. Conde, X-Ray Spectrom. 30, 373 (2001)

26 L.M.P. Fernandes, J.A.M. Lopes, J.M.F. dos Santos, P.E. Knowles, L. Ludhova, F. Mulhauser, F. Kottmann, R. Pohl, D. Taqqu, IEEE Trans. Nucl. Sci. 51, 1575 (2004) 